\title{
Aromatase inhibitors and their use in the sequential setting
}

\author{
R C Coombes, C Harper-Wynne ${ }^{1}$ and M Dowsett ${ }^{1}$ \\ Cancer Research Campaign, Department of Cancer Medicine, Division of Medicine, Imperial College School of \\ Medicine, Charing Cross Hospital, Fulham Palace Road, London W6 8RF, UK \\ ${ }^{1}$ Academic Department of Biochemistry, Royal Marsden Hospital, Fulham Road, London SW3 6JJ, UK \\ (Requests for offprints should be addressed to R C Coombes)
}

\begin{abstract}
Over the past decade several novel aromatase inhibitors have been introduced into clinical practice. The discovery of these drugs followed on from the observation that the main mechanism of action of aminogluthemide was via inhibition of the enzyme aromatase thereby reducing peripheral levels of oestradiol in postmenopausal patients.

The second-generation drug, 4-hydroxyandrostenedione (formestane), was introduced in 1990 and although its use was limited by its need to be given parenterally it was found to be a well-tolerated form of endocrine therapy.

Third-generation inhibitors include vorozole, letrozole, anastrozole and exemestane, the former three being non-steroidal inhibitors, the latter being a steroidal inhibitor. All are capable of inhibiting aromatase action by $>95 \%$ compared with $80 \%$ in the case of 4 -hydroxyandrostenedione.

The sequential use of different generations of aromatase inhibitors in the same patients is discussed. Studies suggest that an optimal sequence of these compounds may well result in longer remission in patients with hormone receptor positive tumours.
\end{abstract}

Endocrine-Related Cancer (1999) 6 259-263

\section{Introduction}

The aromatase enzyme is a cytochrome P450-mediated enzyme complex responsible for the conversion of the androgens androstenedione and testosterone to oestrone and oestradiol respectively. The idea of treating oestrogen-dependent breast cancer with aromatase inhibitors has been around for several years. Aminoglutethimide was the first such compound shown to be clinically effective (Santen et al. 1978). Trials using aminoglutethimide in advanced breast cancer showed that responses occurred in between 20 and $40 \%$ of women so treated and the remission duration ranged from 6 to 12 months (Santen et al. 1978, Harris et al. 1983). A major feature that emerged was the toxicity of aminoglutethimide. Side effects, including skin rash, drowsiness and the inhibition of cortisol synthesis, necessitated the use of corticosteroids with their concomitant side effects. This led to various studies in which lower doses of aminoglutethimide were used in the treatment of patients with breast cancer (Harris et al. 1983, Stuart-Harris et al. 1985). However, the responses seen were generally lower and the duration of response was reduced, although formal trials comparing different dose regimens and using sufficient numbers of patients to provide the necessary statistical power have not been adequately carried out. Before considering the impact of using the drugs in sequence, it is important to consider their effects when given alone.

\section{Second-generation aromatase inhibitors: 4-hydroxyandrostenedione (formestane)}

4-Hydroxyandrostenedione is a compound that has been shown to possess irreversible binding to the enzyme substrate-binding sites (Brodie et al. 1981). This suggests that the compound would cause permanent inactivation and a high degree of selectivity for aromatase. Prolonged inhibition of the enzyme would be likely to result, as recovery of activity is dependent on the rate of neosynthesis of the enzyme. Several studies have been carried out using 4-hydroxyandrostenedione over the past 13 years. Essentially these studies were initially conducted using a lyophilised microcrystalline material and given at a dose of $250 \mathrm{mg}$ every 2 weeks. Peak levels were seen on the first and second day after injection with a fall to less 
than $50 \%$ of peak levels by day 4 . Thereafter the fall in serum 4-hydroxyandrostenedione was approximately loglinear with an apparent half-life of between 5 and 10 days.

4-Hydroxyandrostenedione is principally metabolised to the 4-hydroxyandrostenedione glucuronide which is found in the patients' urine at between 14 and $35 \%$ of the administered dose, and this explains why the drug is rapidly metabolised when administered by the oral route and needs to be administered parenterally. Major routes of metabolism are via dehydrogenation, reduction of the ketone functional groups, reduction of the $\mathrm{C} 4 / \mathrm{C} 5$ double bond and hydroxylation at the C5 position (Poon et al. 1991).

Clinical studies began at the $250 \mathrm{mg}$ dose every 2 weeks (Coombes et al. 1984). This dose selection was based on plasma oestrogen measurements in which we compared the suppression obtained with a single injection of 4-hydroxyandrostenedione at doses of $125-500 \mathrm{mg}$. Comparison of $250 \mathrm{mg}$ and $500 \mathrm{mg}$ showed equivalent degrees of initial suppression but there was an indication of minor recovery in the patients receiving the lower dose. The slightly lower pharmacological effectiveness was confirmed in studies using in vivo aromatisation in which the $250 \mathrm{mg}$ dose reduced peripheral aromatisation to $15.2 \%$ of baseline in comparison with a reduction to $8.1 \%$ with the $500 \mathrm{mg}$ dose $(P<0.05)$ (Jones et al. 1991). Serum oestrone levels measured by gas chromotographic mass spectrometer have been found to fall in parallel with oestradiol levels during intramuscular treatment. The dose of $250 \mathrm{mg}$ every 2 weeks was chosen for clinical studies, as local side effects were much more pronounced for the $500 \mathrm{mg}$ dose.

Other endocrine effects of 4-hydroxyandrostenedione were generally not seen. There are no significant effects on luteinising hormone, follicle-stimulating hormone, sex hormone-binding globulin, dehydroepiandrosterone sulphate, testosterone, 5-dihydrotestosterone or androstenedione when the drug is given intramuscularly. However, when the drug is given orally a small increase in dehydroepiandrosterone sulphate levels is seen. Oral administration of 4-hydroxyandrostenedione is also associated with a dose related fall in sex hormone-binding globulin (Dowsett et al. 1992) and this may be a reflection of the minor androgenic activity associated with the drug.

The drug is clinically very effective (Coombes et al. 1992). The overall complete and partial response rate was $26 \%$ in 136 patients who received therapy for at least 4 weeks. A major determinant of response was oestrogen receptor status of the primary tumour: $93 \%$ of objective responders whose oestrogen receptor was known had oestrogen receptor positive tumours. Prior successful therapy with endocrine therapy was also a determinant: only two of 35 patients who had failed to respond to other endocrine therapy responded. In these studies the drug was well tolerated, with only a minority having any adverse effects. Thus $13 \%$ of patients on the $250 \mathrm{mg}$ dose every 2 weeks complained of some local side effects, principally pain and inflammation at the site of the injection. Only one patient showed androgenic side effects, but anaphylactoid reactions occurred in five patients, presumed to be due to inadvertent intravenous administration.

4-Hydroxyandrostenedione has been given in combination with other endocrine agents. Encouraging results were obtained with a combination of 4-hydroxyandrostenedione and goserelin, the growth hormonereleasing hormone agonist currently used for medical ovariectomy. Goserelin is not able to affect peripheral aromatisation, but, in patients who respond to goserelin and then relapse, the subsequent addition of 4-hydroxyandrostenedione produces further reduction in oestradiol and subsequent response in a high proportion of patients (Stein et al. 1990). Others (Lonning et al. 1992) have also studied the combination of aminoglutethimide and 4-hydroxyandrostenedione in postmenopausal patients with breast cancer. Significant further falls in oestradiol, oestrone and oestrone sulphate were achieved on the addition of aminoglutethimide, with the greatest effect being on oestrone sulphate. Two patients achieved an objective tumour response on the further addition of aminoglutethimide.

4-Hydroxyandrostenedione has also been used in the neoadjuvant setting (Gazet et al. 1996). In this study patients with tumour size (clinical) T1-3 invasive breast cancer were assigned to pretreatment based on oestrogen receptor status. Although patients with hormone receptor negative tumours received chemotherapy, those with oestrogen receptor positive tumours received 4-hydroxyandrostenedione if they were postmenopausal. Treatment was continued for 3 months, and 34 patients received 4-hydroxyandrostenedione with an overall response rate of $35 \%$. At the end of 3 months treatment patients had definitive surgery and/or radiation, depending on the ' $T$ ' stage of the primary tumour. Patients whose disease stabilised or responded to 4-hydroxyandrostenedione continued treatment for a total of 18 months. The 5-year survival rates of this study are the subject of a future paper. This suggests that 4-hydroxyandrostenedione could be used as a neoadjuvant therapy to reduce the size of large or unresectable primary breast carcinomas.

\section{Third-generation aromatase inhibitors}

New aromatase inhibitors include letrozole, anastrozole and vorozole, all of which are non-steroidal aromatase inhibitors, and exemestane, which is an example of a steroidal third-generation aromatase inhibitor. 
Regarding the non-steroidal aromatase inhibitors, several clinical studies have now assessed their efficacy and tolerability. Johnston et al. (1994) studied vorozole at three separate doses (1, 2.5 and $5 \mathrm{mg}$ ) in 24 postmenopausal patients with breast cancer. Increasing doses of vorozole showed greater suppression of oestradiol, oestrone and oestrone sulphate. Eight of 24 patients achieved an objective response: these were principally patients who had responded to tamoxifen. Aside from a slight reduction in serum cortisol, no other endocrine changes were seen.

Vorozole has also been compared with aminoglutethimide in tamoxifen-treated patients. A total of 277 patients received vorozole and 279 patients received aminoglutethimide; responses were seen in 23 and $18 \%$ of patients respectively. Drug-related adverse events were seen in $31 \%$ of patients receiving vorozole and $53 \%$ of patients receiving aminoglutethimide (Bergh et al. 1997). Vorozole has also been compared with megace in tamoxifen-treated patients; complete and partial response rates were seen in $10.5 \%$ of vorozole and $7.6 \%$ of patients receiving megace but weight gain occurred in $14 \%$ of patients receiving megace compared with only $1 \%$ of those receiving vorozole (Goss et al. 1997).

Anastrozole was assessed in an early clinical study (Plourde et al. 1994) and this showed considerable suppression at doses between 1 and $20 \mathrm{mg}$. No other endocrine changes were observed. A study reported by Buzdar et al. (1997) compared anastrozole $1 \mathrm{mg}$, anastrozole $10 \mathrm{mg}$ and megace $160 \mathrm{mg}$ in 386 tamoxifentreated patients. No differences in response rate were seen. However, side effects differed, with weight gain being more prevalent in megace-treated patients and gastrointestinal side effects in anastrozole-treated patients.

In a further study two different doses of letrozole $(0.5$ and $2.5 \mathrm{mg}$ ) were compared with aminoglutethimide at $500 \mathrm{mg}$ daily, and more than 170 patients were accrued in each arm of the study. Complete and partial responses were seen in $16.7 \%$ of the patients receiving the lower dose of letrozole and $17.8 \%$ of those receiving the higher dose, whereas only $11.2 \%$ of aminoglutethimide-treated patients responded. Treatment-related adverse events were seen in 28, 32 and 45\% respectively (Marty et al. 1997).

Exemestane (FCE 24304) was developed some 15 years ago. It causes a time-dependent inactivation of human placental aromatase and has a $K_{\mathrm{i}}$ of $26 \mathrm{nM}$, thus showing an affinity for the enzyme 2.6-fold higher than the substrate androstenedione. In preclinical studies, exemestane induced high rates of regression in Dimethylbenzanthracene (DMBA)-induced mammary tumours in rats $(30 \%$ at $10 \mathrm{mg} / \mathrm{kg}$ and $73 \%$ at $50 \mathrm{mg} / \mathrm{kg}$ ) and these dosages caused an 86 and $93 \%$ decrease in total ovarian aromatase activity respectively (Di Salle et al.
1989, Zaccheo et al. 1991). A phase I endocrine study of exemestane was carried out by Evans et al. (1992). Exemestane was given at doses between 0.5 and $800 \mathrm{mg}$ to groups of between three and four patients. The minimal dose that produced a maximum suppression of plasma oestrogen was $25 \mathrm{mg}$. Although in this study there was evidence that some of the metabolites of exemestane interfered with some of the assays used, oestrone, oestradiol and oestrone sulphate were reduced to 35,28 and $39 \%$ of basal values. No clinically adverse events were attributed to the drug during this study.

Subsequent to this, 27 postmenopausal heavily pretreated patients with metastatic breast cancer were selected for exemestane treatment (Di Salle et al. 1994). Exemestane was given again at between 5 and $600 \mathrm{mg}$ daily to three or four subjects per dose and treatment was given for at least 12 weeks and continued until tumour progression. Minor side effects were seen including dizziness, headaches, hot flushes and nausea. Objective response was seen in eight (two complete responses and six partial responses) of the 24 evaluable patients (33\%; $95 \%$ confidence intervals 18 to $53 \%$ ). Mean duration of response was 42 weeks (12-72 weeks plus). These studies indicated that exemestane was an effective and relatively well-tolerated third-generation aromatase inhibitor, and other subsequent studies have confirmed that the drug is highly active (Di Salle et al. 1994).

\section{Sequential administration of aromatase inhibitors}

We have recently conducted an endocrine study in which patients receiving 4-hydroxyandrostenedione who were responding or stabilising were subsequently given vorozole for a 2 month period (during this time 4-hydroxyandrostenedione was discontinued), and both oestrone and oestradiol were measured sequentially during the course of treatment. The results indicated that the non-steroidal inhibitor vorozole resulted in a further sustained suppression which returned to pretreatment levels once the patients restarted 4-hydroxyandrostenedione treatment (Dowsett et al. 1999).

Previous studies in advanced breast cancer using the non-selective inhibitor aminogluthemide as well as the newer agents have shown that in vivo aromatisation can be inhibited by 85 to $>99 \%$ (Lonning et al. 1991, Jones et al . 1992, Lipton et al. 1995, Masamura et al. 1995, Geisler et al. 1996). As yet, there is no clear correlation between these levels of suppression and clinical efficacy but recent studies suggest that this may be the case as randomised studies using different doses of letrozole have shown improved response rates at higher dosages (Dombernowsky et al. 1998). 
A further study, carried out by our group (Stein et al. 1990), indicated that a stepwise suppression of oestradiol could result in more prolonged benefit for patients with metastatic breast cancer. In this study, premenopausal patients who had achieved either disease stabilisation or remission on goserelin treatment were given 4-hydroxyandrostenedione on relapse and goserelin treatment was maintained. As a result of the introduction of 4-hydroxyandrostenedione, levels of oestradiol fell to similar levels to those observed in postmenopausal patients and the majority of patients obtained a further remission.

We have subsequently studied the clinical effects of giving anastrozole $(1 \mathrm{mg})$ to 21 postmenopausal patients with advanced breast cancer who had already received 4-hydroxyandrostenedione and who had either responded and then relapsed or who had failed to respond. During 4-hydroxyandrostenedione therapy, $10 \%(2 / 21)$ achieved a partial response and $48 \%(10 / 21)$ were classified as stable disease (SD). Nine of these 12 patients showed a further SD on anastrozole with the remaining three showing clear evidence of progression. Seven of these nine patients had oestrogen receptor (ER) positive carcinomas. Four patients achieved SD with anastrozole despite failing to respond to 4-hydroxyandrostenedione. Three of these had ER positive carcinomas. The remaining five patients progressed on both treatments. Five patients stabilised on anastrozole for longer than 6 months, three had progressed on 4-hydroxyandrostenedione (one ER negative) and the other two had stabilised on 4-hydroxyandrostenedione but for less than 6 months (one ER negative). This suggests that sequential reduction in oestradiol in postmenopausal patients may be of benefit.

Several other groups have explored the sequential use of aromatase inhibitors. The administration of 4-hydroxyandrostenedione after aminoglutethimide and exemestane after aminoglutethimide have been reported. Both studies suggest that this approach could be of clinical benefit. The study of Murray \& Pitt (1995) demonstrated that it was possible to obtain responses to 4-hydroxyandrostenedione after aminoglutethimide and these authors observed a $21 \%$ response rate for an average duration of 11 months. The study by Thurlimann et al. (1997), in which 33 aminoglutethimide-resistant patients and 39 aminoglutethimide responders were treated with exemestane $200 \mathrm{mg} / \mathrm{day}$, resulted in a response rate of $12 \%$ and $33 \%$ respectively. This study indicated that nonsteroidal and steroidal aromatase inhibitors are not necessarily cross-resistant. However, the rationale for the former study is different from our 4-hydroxyandrostenedione-anastrozole study as aminoglutethimide and 4-hydroxyandrostenedione, used in the study of Murray \& Pitt (1995), are approximately equipotent in terms of aromatase inhibition. In addition, the study by
Thurlimann et al. (1997) uses a steroidal inhibitor following a non-steroidal inhibitor, i.e. the reverse of the sequence used in the study that we conducted (HarperWynne \& Coombes 1999).

In conclusion, much still needs to be learnt about the correct ways of using aromatase inhibitors. A systematic evaluation of response rates and time to progression with different sequences needs to be undertaken. In addition, randomised comparisons should be made of a stepwise approach to oestrogen degeneration versus immediate maximal degeneration. Only when we have this information will we be able to treat patients rationally in both the adjuvant and advanced settings.

\section{References}

Bergh J, Bonneterre J, Illiger HJ, Murray R, Nortier J, Paridaens R, Rubens RD, Samonigg H \& Van Zyl J for the Vorozole Study Group 1997 Vorozole (Rivizor) versus aminoglutethimide (AG) in the treatment of postmenopausal breast cancer relapsing after tamoxifen. Proceedings of American Society of Clinical Oncology No. 543.

Brodie AMH, Garrett WM, Hendrickson JR, Tsai-Morris CH, Marcotte PA \& Robinson CH 1981 Inactivation of aromatase in vitro by 4-hydroxy-4-androstene-3,17-dione and 4-acetoxy-4-androstene-3,17-dione and sustained effects in vivo. Steroids 38 693-702.

Buzdar AU, Jones SE, Vogel CL, Wolter J, Plourde P \& Webster A for the Arimidex Study Group 1997 A Phase III trial comparing anastrozole ( 1 and 10 milligrams), a potent and selective aromatase inhibitor, with megestrol acetate in postmenopausal women with advanced breast carcinoma. Cancer 79 730-739.

Coombes RC, Goss P, Dowsett M, Gazet J-C \& Brodie A 1984 4-Hydroxyandrostenedione in treatment of postmenopausal patients with advanced breast cancer Lancet 2 1237-1239.

Coombes RC, Hughes SWM \& Dowsett M 1992 4-Hydroxyandrostenedione: a new treatment for postmenopausal patients with breast cancer. European Journal of Cancer 28A 1941 1945.

Di Salle E, Briatico G, Giudici D, Ornati G \& Zaccheo T 1989 Aromatase inhibition and experimental antitumour activity of FCE24304 \& MDL 18962 and SH489. Journal of Steroid Biochemistry 34 431-434.

Di Salle E, Ornati G, Paridaens R, Coombes RC, Lobelle JP \& Zurlo MG 1994 Preclinical and clinical pharmacology of the aromatase inhibitor exemestane (FCE 24304). In Sex Hormones and Antihormones in Endocrine Dependent Pathology: Basic and Clinical Aspects. Eds M Motta \& M Serio. Amsterdam: Elsevier Science B.V .

Dombernowsky P, Smith I, Leonard R, Panasci L, Bellmunt J, Bezwoda W, Gardin G, Gudgeon A, Morgan M, Fornasiero A, Hoffman W, Michel J, Hatscheck T, Tjabbes T, Chaudri HA, Hornberger U \& Trunet PF 1998 Letrozole, a new oral aromatase inhibitor for advanced breast cancer: double-blind randomised trial showing a dose effect and improved efficacy 
and tolerability compared with megestrol acetate. Journal of Clinical Oncology 16 453-461.

Dowsett M, Mehta A, King N, Smith IE, Powles TJ, Stein RC \& Coombes RC 1992 An endocrine and pharmacokinetic study of 4 oral doses of formestane in post-menopausal breast cancer patients. European Journal of Cancer 28 415-420.

Dowsett M, Doody D, Miall S, Howes A, English J \& Coombes RC 1999 Vorozole results in greater oestrogen suppression than foremestane in post-menopausal women and when added to goserelin in pre-menopausal women with advanced breast cancer. Breast Cancer Research and Treatment (In Press).

Evans RTJ, Di Salle E, Ornati G, Lassus M, Benedetti MS, Pianezzola E \& Coombes RC 1992 Phase I and endocrine study of exemestane (FCE24304), a new aromatase inhibitor, in postmenopausal women. Cancer Research 52 5933-5939.

Gazet J-C, Coombes RC, Ford HT, Griffin M, Corbishley C, Makinde V, Lowndes S, Quilliam J \& Sutcliffe R 1996 Assessment of the effect of pretreatment with neoadjuvant therapy on primary breast cancer. British Journal of Cancer 73 758-762.

Geisler J, King N, Dowsett M, Ottestad L, Lundgren S, Walton P, Kormeset PO \& Lonning PE 1996 Influence of anastrozole (Arimidex), a selective, non-steroidal aromatase inhibitor, on in vivo aromatisation and plasma oestrogen levels in postmenopausal women with breast cancer. British Journal of Cancer 74 1286-1291.

Goss P, Wine E, Tannock I, Schwartz IH \& Kremer AB for the North American Vorozole Study Group 1997 Vorozole versus Megace in postmenopausal patients with metastatic breast carcinoma who had relapsed following tamoxifen.

Proceedings of American Society of Clinical Oncology No. 542.

Harper-Wynne C \& Coombes RC 1999 Anastrozole shows evidence of activity in postmenopausal patients who have responded or stabilised on formestane therapy. European Journal of Cancer (In Press)

Harris AL, Dowsett M, Smith IE \& Jeffcoate SL 1983 Endocrine effects of low dose aminoglutethimide alone in advanced postmenopausal breast cancer. British Journal of Cancer 47 621-627.

Johnston SRD, Smith IE, Doody D, Jacobs S, Robertshaw H \& Dowsett M 1994 Clinical and endocrine effects of the oral aromatase inhibitor Vorozole in postmenopausal patients with advanced breast cancer. Cancer Research 54 5875-5881.

Jones AL, MacNeil F, Jacobs S, Lonning PE, Dowsett M \& Powles TJ 1992 The influence of intramuscular 4-hydroxyandrostenedione on peripheral aromatisation in breast cancer patients. European Journal of Cancer 28 17121716.

Lipton A, Demers LM, Harvey HA, Kambic KB, Grossberg H, Brady C, Adkercruetz H, Trunet PF \& Santen RJ 1995 Letrozole (CGS20267). A Phase I study of a new potent oral aromatase inhibitor of breast cancer. Cancer 75 2132-2138.

Lonning PE, Jacobs S, Jones A, Haynes B, Powles T \& Dowsett M 1991 The influence of CGS 16949A on peripheral aromatisation in breast cancer patients. British Journal of Cancer 63 789-793.
Lonning PE, Dowsett M, Jones A, Ekse D, Jacobs S, MacNeill F, Johannessen DC \& Powles TJ 1992 Influence of aminoglutethimide on plasma oestrogen levels in breast cancer patients on 4-hydroxyandrostenedione treatment. Breast Cancer Research and Treatment 23 57-62.

Marty M, Gershanovich M, Campos B, Romieu G, Lurie H, Bonaventura T, Jeffrey M, Buzzi F, Ludwig H, Bodrogi I, Reichardt P, O'Higgins N, Chaudri HA, Friederich P \& Biachoff MA for the Letrozole International Trial Group (AR/ BC3) 1997 Letrozole, a new potent selective aromatase inhibitor (AI) superior to aminoglutethimide (AG) in postmenopausal women with advanced breast cancer (ABC) previously treated with anti-estrogens. Proceedings of American Society of Clinical Oncology No. 544.

Masamura S, Adlercreutz H, Harvey H, Lipton A, Demers LM, Santen RJ \& Santner SJ 1995 Aromatase inhibitor development for treatment for breast cancer. Breast Cancer Research and Treatment 33 19-26.

Murray R \& Pitt P 1995 Aromatase inhibition with 4-OHandrostenedione after prior aromatase inhibition with aminoglutethimide in women with advanced breast cancer. Breast Cancer Research and Treatment 35 249-253.

Plourde OV, Dyroff M \& Dukes M 1994 Arimidex: a potent and selective fourth-generation aromatase inhibitor. Breast Cancer Research and Treatment 30 103-111.

Poon GK, Jarman M, Rowlands MG, Dowsett M \& Firth J 1991 Determination of 4-hydroxyandros-4-ene-3,17-dione metabolism in breast cancer patients using HPLC/MS. Journal of Chromatography 565 75-88.

Santen RJ, Santner S, Davis B, Veldhuis J, Samojlik E \& Ruby E 1978 Aminoglutethimide inhibits extraglandular estrogen production in postmenopausal women with breast carcinoma. Journal of Clinical Endocrinology and Metabolism 47 12571265.

Stein RC, Dowsett M, Hedley A \& Coombes RC 1990 The clinical and endocrine effects of 4-hydroxyandrostenedione alone and in combination with goserelin in premenopausal women with advanced breast cancer. British Journal of Cancer 62 679-683.

Stuart-Harris R, Dowsett M \& D'Souza A 1985 Endocrine effects of low dose aminoglutethimide as an aromatase inhibitor in the treatment of breast cancer. Clinical Endocrinology 22 219-226.

Thurlimann B, Paridaens R, Serin D, Bonneterre J, Roche H, Murray R, di Salle E, Lanzalone S, Zurlo MG \& Piscitelli G 1997 Third-line hormonal treatment with exemestane in postmenopausal patients with advanced breast cancer progressing on aminoglutethimide: a Phase II multicentre multinational study. European Journal of Cancer 33 17671773.

Zaccheo T, Giudici D, Ornati G, Panzeri A \& Di Salle E 1991 Comparison of the effects of the irreversible aromatase inhibitor, exemestane, with atamestane and MDL 18962 in rats with DMBA-induced mammary tumors. European Journal of Cancer 27 1145-1150. 